\title{
Antimicrobial prophylaxis in caesarean section delivery
}

\author{
RONGHUA LIU, LIN LIN and DUJUAN WANG \\ Department of Obstetrics, People's Hospital of Linyi, Linyi, Shandong 276000, P.R. China \\ Received February 8, 2016; Accepted May 17, 2016
}

DOI: $10.3892 /$ etm.2016.3350

\begin{abstract}
Antimicrobial prophylaxis is used routinely for pre-, intra- and post-operative caesarean section. One of the most important risk factors for postpartum infection is caesarean delivery. Caesarean section shows a higher incidence of infection than vaginal delivery. It is complicated by surgical site infections, endometritis or urinary tract infection. The aim of the present study was to assess the usage of antimicrobials in women undergoing caesarean section at a Tertiary Care Hospital. A prospective study was conducted in 100 women during the period of February 2013 to August 2013 in the inpatient Department of Gynaecology and Obstetrics. Data collected included the age of the patient, gravidity, and type of caesarean section, which was analyzed for the nature and number of antimicrobials prescribed, duration of treatment, polypharmacy, fixed-dose combinations, generic/brand names used and failure of prophylaxis. Antimicrobial prophylaxis was administered to the patients. The most commonly prescribed antimicrobial was a combination of ceftriaxone and sulbactam. Of 100 patients, 87\% were aged 20-35 years. The highest proportion of patients were primigravida $72 \%$. Elective procedure was carried out in $38 \%$, the remaining were emergency $\mathrm{C}$-section in whom intra- and post-operative antimicrobial prophylaxis was given for a duration of 7 days. In total, $27 \%$ of patients were reported with infection even after the antimicrobial prophylaxis. In conclusion, pre-operative prophylaxis was given in the early rupture of membranes. Fixed-dose combinations were preferred. Incidence of infection even after antimicrobial prophylaxis was reported due to pre-existing infection, debilitating disease or prolonged rupture of membranes. Patients with recurrent infection were shifted to amoxicillin and clavulinic acid combination. Drugs were prescribed only by brand names which is of concern.
\end{abstract}

Correspondence to: Dr Lin Lin, Department of Obstetrics, People's Hospital of Linyi, 27 Jiefang Road, Linyi, Shandong 276000, P.R. China

E-mail: linlin1507@126.com

Key words: antimicrobial prophylaxis, caesarean section, drug utilization, surgical site infections

\section{Introduction}

Antimicrobials are commonly prescribed in Obstetrics and Gynaecology prophylactically for pre-operative and postoperative procedures (1) (caesarean section, episiotomy, medical termination of pregnancy, total vaginal or abdominal hysterectomy, laparoscopic procedures, tubal ligation, dilatation and curettage, and myomectomy) or to treat ongoing infection (vaginitis, pelvic inflammatory disease, endometriosis, sexually transmitted diseases and urinary tract infections).

The rational use of antimicrobials in women of the child-bearing age group is important because it affects this population as well as their offspring. Indiscriminate use of antibiotics may result in the appearance of drug-resistant organisms (2).

Drug utilization is defined by the WHO as 'The marketing, distribution, prescription, and use of drugs in society, with special emphasis on the resulting medical, social, and economic consequences'. A drug utilization study is therefore one designed to describe quantitatively and qualitatively the use of a given drug, i.e., class of drugs, indications, duration of treatment, dosage, previous or associated treatments and compliance $(3,4)$.

Surgical site infections are a common complication of obstetric and gynaecological procedures. The use of antimicrobial prophylaxis for caesarean section has been shown to be effective in reducing postoperative morbidity, cost and duration of hospitalization (5-8).

Antimicrobial usage in the above setting becomes inevitable but should be restricted. The judicious use of these drugs can prevent post-partum infection of the mother and neonate and reduce incidence of adverse drug reactions.

The present study was performed to assess the usage of antimicrobials in 100 women undergoing caesarean section

\section{Materials and methods}

A prospective study of 100 prescriptions of women attending the inpatient and outpatient Department of Obstetrics and Gynaecology, People's Hospital of Linyi (Shandong, China), a Tertiary Care Hospital during the period of February 2013 to August 2013 was conducted. Prior to start of the study Institutional Ethics Committee approval was obtained. The prescriptions were analyzed using WHO core drug use indicators including average number of drugs per encounter, percentage of drugs prescribed by generic name, percentage of 


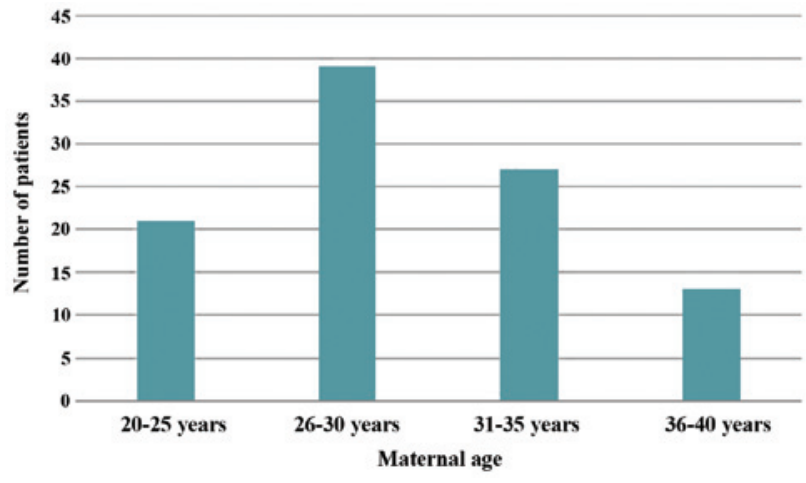

Figure 1. Maternal age at delivery. Highest proportions of patients were primigravida $72 \%$. Elective caesarean section was carried out in $38 \%$, and the remaining were emergency cases. Intra- and post-operative antimicrobial prophylaxis was given in both settings of patients for a duration of 7-12 days. Intra-operative prophylaxis was associated in patients with long duration of rupture of membranes. Patients who underwent the elective procedure received pre-operative antimicrobial prophylaxis.

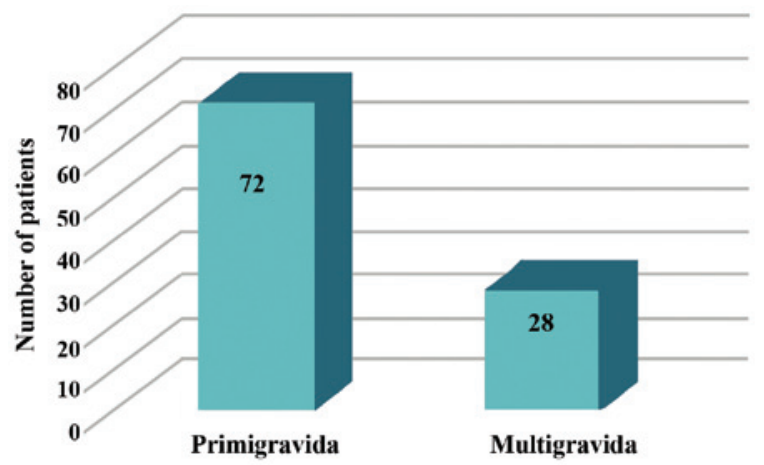

Figure 2. Type of gravida. Antimicrobial prophylaxis was prescribed to all the patients. The total number of antimicrobials prescribed post-operatively, during hospital stay, discharge and after suture removal ranged between 4 and 8 (average 5).

drugs prescribed from the essential drug list, patient care and availability of drugs (4).

The data collected were analyzed for the following parameters: i) age of patient; ii) gravidity; iii) type of caesarean section - (elective/emergency); iv) number of drugs per prescription; v) class of antimicrobials; vi) duration of antimicrobial treatment; vii) average number of antimicrobials prescribed; viii) polypharmacy; ix) number of fixed-dose combinations prescribed; $x$ ) generic/brand name prescribed; and xi) failure of prophylaxis.

\section{Results}

Of 100 patients, $87 \%$ were in the age group of $20-35$ years, $21 \%$ were in the age group of $20-25$ years, $39 \%$ were in the age group of 26-30 years, $27 \%$ were in the age group of 31-35 years and the remaining 13\% were in the age group of 36-39 years (Fig. 1). The highest proportion of patients was primigravida (72\%) (Fig. 2). Elective C-sections were carried out in $38 \%$ of cases and the remaining were emergency $\mathrm{C}$-section in whom intra- and post-operative antimicrobial prophylaxis was received for a duration of 7 days (Fig. 3). Antimicrobial prophylaxis was given to all the patients, while the most commonly

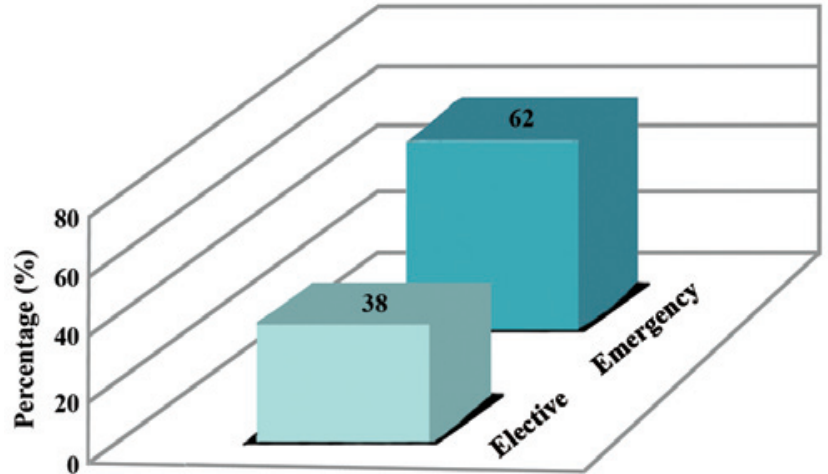

Figure 3. Type of C-section. The most commonly prescribed antibiotics were ceftriaxone, sulbactam, amikacin, metronidazole, satranidazole, amoxicillin, clavulanic acid, ciprofoxacin and tinidazole. The most commonly prescribed fixed-dose combinations were the ceftriaxone and sulbactam combination, amoxicillin and clavulinic acid combination and ciprofoxacin and tinidazole combination. A total of 214 fxed-dose combinations were prescribed. The prescriptions were prescribed by brand names.

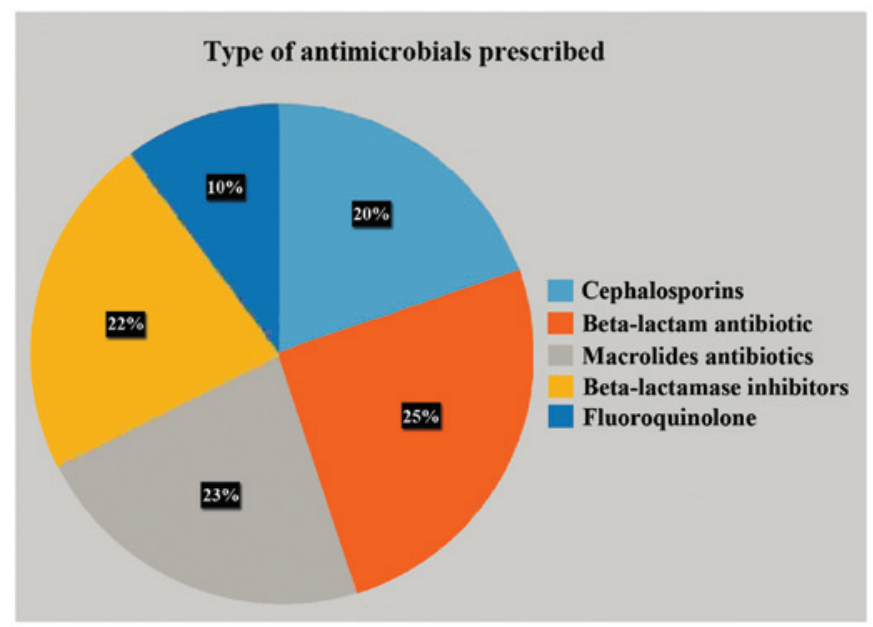

Figure 4. Class of antimicrobials prescribed.

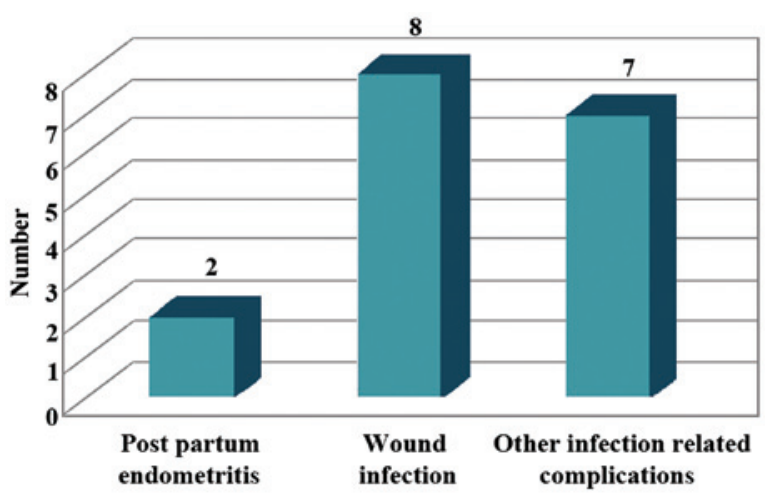

Figure 5. Complications due to failure of antibiotic prophylaxis.

prescribed antimicrobial was a combination of ceftriaxone and sulbactam (Fig. 4). Patients who were reported with infection even after antimicrobial prophylaxis were $27 \%$ (Fig. 5).

Post-partum endometritis develops usually in patients undergoing caesarean section, after labour or rupture of 
Table I. Number of antimicrobials prescribed per patient.

\begin{tabular}{lc} 
No. of antimicrobials & Percentage of use \\
\hline 4 & 6 \\
5 & 22 \\
6 & 28 \\
7 & 13 \\
8 & 16 \\
9 or more & 15 \\
\hline
\end{tabular}

Table II. Antepartum complications.

\begin{tabular}{lc}
\hline Antepartum complications & No. of patients \\
\hline Antepartum infections & 17 \\
Gestational diabetes & 5 \\
Antepartum anemia & 44 \\
\hline
\end{tabular}

membranes despite the use of prophylactic antibiotics (9). Therapeutic use of antimicrobials was 'empirical'. Patients who were reported with infection even after antimicrobial prophylaxis were $17 \%$ of which $2 \%$ were diagnosed with post-partum endometritis, $8 \%$ with wound infection and $7 \%$ with urinary tract infection $(10,11)$. The patients with failure of antimicrobial prophylaxis were shifted to other regimens following empiric treatment (Tables I and II).

\section{Discussion}

The largest number of patients in the present study was in the age group of 26-30 years. The age distribution is a reflection of the demographic status of the community as the majority of women tend to complete their families during this period. The emergency lower segment caesarean section (LSCS) group formed over $50 \%$ of the study participants. It is important to opt for elective procedures, where feasible, as it provides the opportunity to have a clean operative field $(12,13)$. LSCS were performed under antimicrobial prophylaxis to avoid post-operative morbidity. This reflects that, in general, the practice in the audit setting is evidence-based.

Regardless of the antibiotic regimen used, the protective effect of prophylactic antibiotics was homogeneous across all the patients undergoing caesarean section. This effect of significant reduction in postoperative infectious morbidity (by around two thirds) led the reviewers to recommend that antibiotic prophylaxis be provided to all the women undergoing caesarean section. All the patients received antimicrobial prophylaxis, which is good clinical practice and comparable to international standards of evidence-based practice worldwide. The reduction in post-operative infection-related morbidity, in turn, is cost effective as it reduces the number of days the patient has to stay in hospital. Additionally, it reduces the incidence of hospital-acquired infections and infection with more resistant microorganisms.
The most frequent microbes isolated from endometrial cultures of women with post-caesarean wound infections are staphylococci, enterococci, anaerobes and ureaplasmas (14-17). Furthermore, when specifically identified, ureaplasma (or Mycoplasma genus) is the most common organism isolated from the amniotic fluid and chorioamnion at caesarean delivery, and is associated with a 3- to 8-fold increased risk of post-caesarean endometritis or wound infection $(18,19)$. Bacterial vaginosis is also associated with an increased risk of post-caesarean endometritis. Therefore, the recommended regimen of ceftriaxone alone does not cover frequent isolates or risk factors of such infection. Thus, use of an extended spectrum regimen involving a second antibiotic metronidazole was used $(9,20,21)$.

In conclusion, antimicrobial prophylaxis was given routinely to all 100 patients undergoing caesarean section pre-operatively, intra-operatively and post-operatively. Use of prophylactic antibiotics for patients undergoing caesarean section has significantly reduced post-operative maternal infection, morbidity and hospital stay $(22,23)$. Pre-operative prophylaxis was given in the early rupture of membranes. Fixed-dose combinations were used, the most common being ceftriaxone and sulbactam combination. Incidence of infection even after antimicrobial prophylaxis was reported due to pre-existing infection, debilitating disease or prolonged rupture of membranes. Patients with recurrent infection were shifted to a regime that included amoxicillin and clavulanic acid, ciprofloxacin and tinidazole combination and the duration of treatment was prolonged (24). Patients with failure of antimicrobial prophylaxis were treated following the change in antimicrobial regimen, prolonging the duration of treatment and dilatation and curettage in $2 \%$ of the patients who reported with post-partum endometritis. Drugs were prescribed only by brand names which is of concern, generic drug prescribing is recommended and would enhance cost-effectiveness. Use of certain antimicrobials (fluoroquinolones and nitro-imidazole) not recommended in lactating mothers could have been avoided.

Since the patients with failure of antimicrobial prophylaxis received 'empiric' treatment, antibiotic culture sensitivity testing is recommended prior to introducing a change in the antimicrobial treatment regimen, to exclude antibiotic resistance.

\section{References}

1. Warnecke HH, Graeff H, Selbmann HK, Preac-Mursic V, Adam D, Gloning KP, Jänicke F and Zander J: Perioperative short-term prophylaxis with antibiotics in caesarean section. Geburtshilfe Frauenheilkd 42: 654-661, 1982 (In German).

2. Smaill FM and Grivell RM: Antibiotic prophylaxis versus no prophylaxis for preventing infection after cesarean section. Cochrane Database Syst Rev 10: CD007482, 2014.

3. de Jonge L, Bos HJ, van Langen IM, de Jong-van den Berg LT and Bakker MK: Antibiotics prescribed before, during and after pregnancy in the Netherlands: a drug utilization study. Pharmacoepidemiol Drug Saf 23: 60-68, 2014.

4. Lee F, Wong P, Hill F, Burgner D and Taylor R: Evidence behind the WHO guidelines: hospital care for children: what is the role of prophylactic antibiotics in the management of burns? J Trop Pediatr 55: 73-77, 2009.

5. Clifford V and Daley A: Antibiotic prophylaxis in obstetric and gynaecological procedures: a review. Aust N Z J Obstet Gynaecol 52: 412-419, 2012.

6. van Schalkwyk J, Van Eyk N, Yudin MH, Boucher M, Cormier B Gruslin A, Money DM, Ogilvie G, Castillo E, Paquet C, et al; Society of Obstetricians and Gynaecologists of Canada Infectious Diseases Committee: Antibiotic prophylaxis in obstetric procedures. J Obstet Gynaecol Can 32: 878-892, 2010. 
7. Janisch H, Philipp K and Riss P: The effect of antibiotic prophylaxis in vaginal obstetric procedures (author's transl) Wien Klin Wochenschr 91: 227-230, 1979 (In German).

8. Tita AT, Rouse DJ, Blackwell S, Saade GR, Spong CY and Andrews WW: Emerging concepts in antibiotic prophylaxis for cesarean delivery: a systematic review. Obstet Gynecol 113: 675-682, 2009.

9. Watts DH, Krohn MA, Hillier SL and Eschenbach DA: Bacterial vaginosis as a risk factor for post-cesarean endometritis. Obstet Gynecol 75: 52-58, 1990.

10. Mara JE and Baker JL Jr: Hypertension and haematomas: prophylaxis with apresoline. Br J Plast Surg 30: 169-170, 1977.

11. Lamont RF, Sobel JD, Kusanovic JP, Vaisbuch E, Mazaki-Tovi S, Kim SK, Uldbjerg N and Romero R: Current debate on the use of antibiotic prophylaxis for caesarean section. BJOG 118: 193-201, 2011.

12. Dlamini LD, Sekikubo M, Tumukunde J, Kojjo C, Ocen D, Wabule A and Kwizera A: Antibiotic prophylaxis for caesarean section at a Ugandan hospital: a randomised clinical trial evaluating the effect of administration time on the incidence of postoperative infections. BMC Pregnancy Childbirth 15: 91, 2015.

13. Liabsuetrakul T, Lumbiganon $\mathrm{P}$ and Chongsuvivatwong V: Prophylactic antibiotic prescription for cesarean section. Int J Qual Health Care 14: 503-508, 2002.

14. Newton ER and Wallace PA: Effects of prophylactic antibiotics on endometrial flora in women with postcesarean endometritis. Obstet Gynecol 92: 262-268, 1998.

15. Sherman D, Lurie S, Betzer M, Pinhasi Y, Arieli S and Boldur I: Uterine flora at cesarean and its relationship to postpartum endometritis. Obstet Gynecol 94: 787-791, 1999.
16. Watts DH, Eschenbach DA and Kenny GE: Early postpartum endometritis: the role of bacteria, genital mycoplasmas, and Chlamydia trachomatis. Obstet Gynecol 73: 52-60, 1989.

17. Emmons SL, Krohn M, Jackson M and Eschenbach DA: Development of wound infections among women undergoing cesarean section. Obstet Gynecol 72: 559-564, 1988.

18. Roberts S, Maccato M, Faro S and Pinell P: The microbiology of post-cesarean wound morbidity. Obstet Gynecol 81: 383-386, 1993.

19. Andrews WW, Shah SR, Goldenberg RL, Cliver SP, Hauth JC and Cassell GH: Association of post-cesarean delivery endometritis with colonization of the chorioamnion by Ureaplasma urealyticum. Obstet Gynecol 85: 509-514, 1995

20. Keski-Nisula L, Kirkinen P, Katila ML, Ollikainen M, Suonio S and Saarikoski S: Amniotic fluid U. urealyticum colonization: significance for maternal peripartal infections at term. Am J Perinatol 14: 151-156, 1997.

21. Yoon BH, Romero R, Park JS, Chang JW, Kim YA, Kim JC and Kim KS: Microbial invasion of the amniotic cavity with Ureaplasma urealyticum is associated with a robust host response in fetal, amniotic, and maternal compartments. Am J Obstet Gynecol 179: 1254-1260, 1998.

22. Gordon HR, Phelps D and Blanchard K: Prophylactic cesarean section antibiotics: maternal and neonatal morbidity before or after cord clamping. Obstet Gynecol 53: 151-156, 1979.

23. Itskovitz J, Paldi E and Katz M: The effect of prophylactic antibiotics on febrile morbidity following cesarean section. Obstet Gynecol 53: 162-165, 1979.

24. Ledger WJ: Prophylactic antibiotics in cesarean section. Am J Obstet Gynecol 194: 1500, 2006. 